\title{
INVERSIVE CONGRUENTIAL PSEUDORANDOM NUMBERS AVOID THE PLANES
}

\author{
JÜRGEN EICHENAUER-HERRMANN
}

\begin{abstract}
Nonlinear congruential pseudorandom number generators based on inversions have recently been introduced and analyzed. These generators do not show the lattice structure of the widely used linear congruential method. In the present paper it is proved that the points formed by $d$ consecutive pseudorandom numbers of an inversive congruential generator with prime modulus possess an even stronger property: Any hyperplane in $d$-space contains at most $d$ of these points, that is to say, the hyperplane spanned by $d$ arbitrary points of an inversive congruential generator contains no further points. This feature makes the inversive congruential method particularly attractive for simulation problems where linear structures within the generated points should be avoided.
\end{abstract}

\section{INTRODUCTION}

The well-known lattice structure of linear congruential pseudorandom numbers makes them too regular for certain simulation purposes [2]. This defect of the linear congruential method was first pointed out by G. Marsaglia in his famous paper Random numbers fall mainly in the planes [4]. Therefore, nonlinear congruential pseudorandom number generators, which do not show the undesirable lattice structure, have been proposed and studied recently (cf. [1, 2, $3,5,6,7])$. In the present paper a nonlinear congruential generator based on inversions with respect to a prime modulus is considered.

Let $p \geq 3$ be a prime number, and denote by $\mathbb{Z}_{p}=\{0,1, \ldots, p-1\}$ and $\mathbb{Z}_{p, 1}=\{1,2, \ldots, p-1\}$ the set of nonnegative and the set of positive integers less than $p$, respectively. For an integer $x \in \mathbb{Z}_{p, 1}$, let $x^{-1}$ be the multiplicative inverse of $x$ modulo $p$. For integers $a, b \in \mathbb{Z}_{p, 1}$ an inversive congruential sequence $\left(x_{n}\right)_{n \geq 0}$ in $\mathbb{Z}_{p}$ is obtained by the recursion

$$
x_{n+1} \equiv\left\{\begin{array}{ll}
a x_{n}^{-1}+b(\bmod p) & \text { for } x_{n} \neq 0, \\
b & \text { for } x_{n}=0,
\end{array} \quad n \geq 0 .\right.
$$

In [2], conditions are derived for the generated sequence to have maximal period length $p$, which we assume to be true from now on. For example, the sequence has period length $p$ if $x^{2}-b x-a$ is a primitive polynomial over the finite field $\mathbb{Z}_{p}$.

Received November 30, 1989.

1980 Mathematics Subject Classification (1985 Revision). Primary 65C10: Secondary 11 K45. 
Let $d$ be an integer with $2 \leq d<p$, and denote by

$$
V_{d}=\left\{\left(x_{n}, \ldots, x_{n+d-1}\right) \in \mathbb{Z}_{p}^{d} \mid x_{n}, \ldots, x_{n+d-2} \neq 0,0 \leq n<p\right\}
$$

the set of $d$-tuples of consecutive pseudorandom numbers generated by the inversive congruential method, where those $d$-tuples are omitted which contain a zero in one of the first $d-1$ coordinates. These $d-1$ "boundary" points are excluded for the sake of simplicity. For arbitrary integers $\alpha_{0}, \alpha_{1}, \ldots, \alpha_{d} \in \mathbb{Z}_{p}$ with $\left(\alpha_{1}, \ldots, \alpha_{d}\right) \neq(0, \ldots, 0)$ the set

$$
H=\left\{\left(z_{1}, \ldots, z_{d}\right) \in \mathbb{Z}_{p}^{d} \mid \alpha_{1} z_{1}+\cdots+\alpha_{d} z_{d} \equiv \alpha_{0}(\bmod p)\right\}
$$

is a hyperplane in $\mathbb{Z}_{p}^{d}$. The main result of the present paper, which is proved in $\S 3$, is given in the following theorem.

Theorem. Any hyperplane $H$ in $\mathbb{Z}_{p}^{d}$ contains at most $d$ points of the set $V_{d}$.

This result demonstrates that, in contrast to the linear congruential method, inversive congruential pseudorandom numbers do not fall in the planes, they even "avoid" concentrating on any hyperplane. Therefore, the inversive congruential method seems to be particularly suitable for simulation problems where linear structures within the generated points may influence the simulation outcome, e.g., in simulating certain geometric probabilities.

\section{AuXiliary Results}

First, some further notation is necessary. Put $\mathbb{Z}_{p, 0}=\mathbb{Z}_{p}$. A function $f_{1}: \mathbb{Z}_{p, 1} \rightarrow \mathbb{Z}_{p}$ is given by

$$
f_{1}(x) \equiv a x^{-1}+b \quad(\bmod p),
$$

and for $k$ with $2 \leq k<p$, sets $\mathbb{Z}_{p, k}$ and functions $f_{k}: \mathbb{Z}_{p, k} \rightarrow \mathbb{Z}_{p}$ are defined recursively by

$$
\mathbb{Z}_{p, k}=\left\{x \in \mathbb{Z}_{p, k-1} \mid f_{k-1}(x) \neq 0\right\}
$$

and

$$
f_{k}(x)=f_{1}\left(f_{k-1}(x)\right),
$$

respectively. Let $\pi_{0}: \mathbb{Z}_{p} \rightarrow \mathbb{Z}_{p}$ with $\pi_{0}(x)=x$ be the identity on $\mathbb{Z}_{p}$, and for $k$ with $1 \leq k<p$, define functions $\pi_{k}: \mathbb{Z}_{p, k} \rightarrow \mathbb{Z}_{p}$ by

$$
\pi_{k}(x) \equiv x \prod_{j=1}^{k} f_{j}(x) \quad(\bmod p) .
$$

A linear congruential sequence $\left(\tau_{n}\right)_{n \geq 0}$ in $\mathbb{Z}_{p}$ is given by $\tau_{0}=0, \tau_{1}=1$, and

$$
\tau_{n} \equiv b \tau_{n-1}+a \tau_{n-2}(\bmod p), \quad n \geq 2,
$$

and for $k$ with $0 \leq k<p$, linear functions $l_{k}: \mathbb{Z}_{p} \rightarrow \mathbb{Z}_{p}$ are defined by

$$
l_{k}(x) \equiv \tau_{k+1} x+\tau_{k} a \quad(\bmod p) .
$$


Lemma 1. Let $0 \leq k<p$. The function $\pi_{k}$ is the restriction of the linear function $l_{k}$ to the set $\mathbb{Z}_{p, k}$, i.e., $\pi_{k}(x)=l_{k}(x)$ for $x \in \mathbb{Z}_{p, k}$.

Proof. The lemma is proved by induction on $k$. For $k=0$ the assertion follows from $\tau_{0}=0$ and $\tau_{1}=1$. If it is valid for some integer $k$ with $0 \leq k<p-1$, then

$$
\begin{aligned}
\pi_{k+1}(x) & \equiv x \pi_{k}\left(f_{1}(x)\right) \equiv x l_{k}\left(f_{1}(x)\right) \\
& \equiv x\left(\tau_{k+1} f_{1}(x)+\tau_{k} a\right) \\
& \equiv x\left(\tau_{k+1}\left(a x^{-1}+b\right)+\tau_{k} a\right) \\
& \equiv\left(b \tau_{k+1}+a \tau_{k}\right) x+\tau_{k+1} a \\
& \equiv \tau_{k+2} x+\tau_{k+1} a \equiv l_{k+1}(x) \quad(\bmod p)
\end{aligned}
$$

for $x \in \mathbb{Z}_{p, k+1}$, which completes the proof. with

It follows from the results in [2] that $\tau_{1}, \ldots, \tau_{p} \neq 0$. Therefore, $\xi_{k} \in \mathbb{Z}_{p}$

$$
\xi_{k} \equiv-\tau_{k+1}^{-1} \tau_{k} a \quad(\bmod p)
$$

is the unique zero of the linear function $l_{k}$ for $0 \leq k<p$.

Lemma 2. The zeros $\xi_{0}, \ldots, \xi_{p-1}$ of the linear functions $l_{0}, \ldots, l_{p-1}$ are pairwise different, i.e., $\left\{\xi_{0}, \ldots, \xi_{p-1}\right\}=\mathbb{Z}_{p}$.

Proof. For $1 \leq k \leq p$, define integers $y_{k} \in \mathbb{Z}_{p}$ by $y_{k} \equiv \tau_{k+1} \tau_{k}^{-1}(\bmod p)$. Then

$$
\begin{aligned}
y_{k+1} & \equiv\left(b \tau_{k+1}+a \tau_{k}\right) \tau_{k+1}^{-1} \\
& \equiv a y_{k}^{-1}+b(\bmod p), \quad 1 \leq k<p,
\end{aligned}
$$

i.e., $y_{1}, \ldots, y_{p}$ are the first $p$ elements of an inversive congruential sequence with maximal period length $p$, which implies that $\left\{y_{1}, \ldots, y_{p}\right\}=\mathbb{Z}_{p}$. Because of

$$
\xi_{k} \equiv b-y_{k+1}(\bmod p), \quad 0 \leq k<p,
$$

the integers $\xi_{0}, \ldots, \xi_{p-1}$ are pairwise different.

For arbitrary integers $\alpha_{0}, \alpha_{1}, \ldots, \alpha_{d} \in \mathbb{Z}_{p}$ with $\left(\alpha_{1}, \ldots, \alpha_{d}\right) \neq(0, \ldots, 0)$, a polynomial $P_{d}: \mathbb{Z}_{p} \rightarrow \mathbb{Z}_{p}$ is defined by

$$
P_{d}(x) \equiv\left(\alpha_{1} x-\alpha_{0}\right) \prod_{j=0}^{d-2} l_{j}(x)+\sum_{k=2}^{d} \alpha_{k} l_{k-1}(x) \prod_{\substack{j=0 \\ j \neq k-2}}^{d-2} l_{j}(x) \quad(\bmod p) .
$$

Lemma 3. The polynomial $P_{d}$ has at most $d$ zeros.

Proof. First, we prove by contradiction that the polynomial $P_{d}$ is not identically zero. Indeed, assume that $P_{d}(x)=0$ for every $x \in \mathbb{Z}_{p}$. Since $\xi_{i}$ is the zero of the linear function $l_{i}$, it follows that

$$
P_{d}\left(\xi_{i}\right) \equiv \alpha_{i+2} l_{i+1}\left(\xi_{i}\right) \prod_{\substack{j=0 \\ j \neq i}}^{d-2} l_{j}\left(\xi_{i}\right) \quad(\bmod p)
$$


for $0 \leq i \leq d-2$. Hence, the assumption $P_{d}\left(\xi_{i}\right)=0$ and Lemma 2 imply that $\alpha_{i+2}=0$ for $0 \leq i \leq d-2$ which yields

$$
P_{d}(x) \equiv\left(\alpha_{1} x-\alpha_{0}\right) \prod_{j=0}^{d-2} l_{j}(x) \quad(\bmod p)
$$

for $x \in \mathbb{Z}_{p}$. Therefore, it follows from the assumption $P_{d}\left(\xi_{d-1}\right)=P_{d}\left(\xi_{d}\right)=0$ and Lemma 2 that $\alpha_{1}=0$, which contradicts $\left(\alpha_{1}, \ldots, \alpha_{d}\right) \neq(0, \ldots, 0)$ and shows that the polynomial $P_{d}$ is not identically zero. This proves the lemma, since $P_{d}$ is at most of degree $d$.

\section{Proof of THE THEOREM}

The set $V_{d}$ can be written in the form

$$
V_{d}=\left\{\left(x, f_{1}(x), \ldots, f_{d-1}(x)\right) \in \mathbb{Z}_{p}^{d} \mid x \in \mathbb{Z}_{p, d-1}\right\},
$$

since the inversive congruential sequence $\left(x_{n}\right)_{n \geq 0}$ has maximal period length $p$. Therefore,

$$
\begin{aligned}
\#\left(H \cap V_{d}\right) & =\#\left\{x \in \mathbb{Z}_{p, d-1} \mid \alpha_{1} x+\alpha_{2} f_{1}(x)+\cdots+\alpha_{d} f_{d-1}(x) \equiv \alpha_{0}(\bmod p)\right\} \\
& =\#\left\{x \in \mathbb{Z}_{p, d-1} \mid\left(\alpha_{1} x+\alpha_{2} f_{1}(x)+\cdots+\alpha_{d} f_{d-1}(x)\right) \prod_{j=0}^{d-2} \pi_{j}(x)\right. \\
& =\#\left\{x \in \mathbb{Z}_{p, d-1} \mid\left(\alpha_{1} x-\alpha_{0}\right) \prod_{j=0}^{d-2} \pi_{j}(x)\right. \\
+\sum_{k=2}^{d} \alpha_{k} f_{k-1}(x) \pi_{k-2}(x) & \left.\prod_{\substack{j=0 \\
j \neq k-2}}^{d-2} \pi_{j}(x) \equiv 0(\bmod p)\right\} \\
& =\#\left\{\begin{array}{c}
x \in \mathbb{Z}_{p, d-1} \mid\left(\alpha_{1} x-\alpha_{0}\right) \prod_{j=0}^{d-2} \pi_{j}(x) \\
+\sum_{k=2}^{d} \alpha_{k} \pi_{k-1}(x) \prod_{\substack{j=0 \\
j \neq k-2}}^{d-2} \pi_{j}(x) \equiv 0(\bmod p)
\end{array}\right\}
\end{aligned}
$$

for any hyperplane $H$ in $\mathbb{Z}_{p}^{d}$. Now, Lemmas 1 and 3 show that

$$
\#\left(H \cap V_{d}\right)=\#\left\{x \in \mathbb{Z}_{p, d-1} \mid P_{d}(x)=0\right\} \leq d,
$$

which completes the proof of the theorem. 


\section{BIBLIOGRAPHY}

1. J. Eichenauer, H. Grothe, and J. Lehn, Marsaglia's lattice test and non-linear congruential pseudo random number generators, Metrika 35 (1988), 241-250.

2. J. Eichenauer and J. Lehn, A non-linear congruential pseudo random number generator, Statist. Papers 27 (1986), 315-326.

3. J. Eichenauer, J. Lehn, and A. Topuzoğlu, A nonlinear congruential pseudorandom number generator with power of two modulus, Math. Comp. 51 (1988), 757-759.

4. G. Marsaglia, Random numbers fall mainly in the planes, Proc. Nat. Acad. Sci. U.S.A. 61 (1968), 25-28.

5. H. Niederreiter, Lower bounds for the discrepancy of inversive congruential pseudorandom numbers, Math. Comp. 55 (1990), 277-287.

6. __ Remarks on nonlinear congruential pseudorandom numbers, Metrika 35 (1988), 321328.

7. $\ldots$, The serial test for congruential pseudorandom numbers generated by inversions, Math. Comp. 52 (1989), 135-144.

Fachbereich Mathematik, Technische Hochschule, Schlossgartenstrasse 7, D-6100 Darmstadt, Federal Republic of Germany 